\title{
ON THE SUMMABILITY OF FOURIER SERIES. I*
}

\author{
BY \\ EINAR HILLE AND J. D. TAMARKIN
}

1. Introduction. The present paper is the first of a sequence of memoirs that the authors intend to devote to various aspects of the theory of summability of Fourier series and of associated series. In this first paper we consider the application of the Nörlund means to such series, and we derive sufficient (partly also necessary) conditions that such a method of summation be effective with respect to these series in one sense or other to be described below.

Let $A$ denote a regular method of limitation (summation) defined by a triangular† matrix $\mathfrak{A}=\left\|a_{m n}\right\|$, and the infinite set of equations

$$
y_{m}=\sum_{n=0}^{m} a_{m n} x_{n} \quad(m=0,1,2, \cdots)
$$

which coördinate a new sequence $\left\{y_{m}\right\}$ to a given sequence $\left\{x_{n}\right\}$. The assumed regularity of $\mathfrak{A}$ implies that $\lim y_{m}=\lim x_{n}$ whenever the latter limit exists as a finite quantity. The sequence $\left\{x_{n}\right\}$ is limitable $A$ to the limit $\xi$ if $\lim y_{m}=\xi$. If $x_{n}$ denotes the $n$th partial sum of a series, and lim $y_{m}=\xi$, the series is said to be summable $A$ to the sum $\xi$. We denote by $A$ the class of all regular triangular matrices $\mathfrak{A}$.

We shall be concerned with a sub-class $\mathcal{N}$ of $\mathcal{A}$ consisting of matrices $\Re$. Each matrix $\mathfrak{N}$ is defined by a sequence of complex numbers $\left\{p_{v}\right\}$ such that

$$
P_{n} \equiv p_{0}+p_{1}+\cdots+p_{n} \neq 0 .
$$

The elements $a_{m n}$ of $\mathfrak{N}$ are defined by

$$
a_{m n}=\left\{\begin{array}{cc}
p_{m-n} / P_{m}, & n \leqq m \\
0, & n>m,
\end{array}\right.
$$

so that the generalized limit of the sequence $\left\{x_{n}\right\}$ is taken to be

$$
\left(N, p_{n}\right)-\lim x_{n}=\lim _{n \rightarrow \infty} P_{n}^{-1}\left(p_{n} x_{0}+p_{n-1} x_{1}+\cdots+p_{0} x_{n}\right)
$$

* Presented to the Society, September 7, 1928, and March 25, 1932; received by the editors April 19, 1932. Some special cases of the results of the present paper were communicated to the National Academy of Sciences on November 7, 1928, see [4]. Here and below numbers in square brackets refer to the Bibliography at the end of the paper.

$\dagger$ The matrix is of course an infinite square matrix. The word triangular refers to the fact that all the elements above the main diagonal are zero: $a_{m n}=0$ if $n>m$. 
provided this limit exists. The conditions of regularity are

$$
\sum_{k=0}^{n}\left|p_{k}\right|<C\left|P_{n}\right|, \quad p_{n} / P_{n} \rightarrow 0,
$$

where $C$ denotes a fixed positive constant.

Such a definition of limitation was first given by G. F. Woronoi [8] who assumed that $p_{n}>0$ and that $n^{-\alpha} P_{n}$ is bounded for some value of $\alpha$. Woronoi's note was scarcely observed at the time of its appearance and was, at any rate, soon forgotten. It is customary nowadays to attach these definitions of summation to the name of N. E. Nörlund [6] who proved some important properties of such means assuming $p_{n}>0$ and $p_{n} / P_{n} \rightarrow 0$. We conform to this usage in the following. We use the symbol $\left(N, p_{v}\right)$ to denote the Nörlund method of summation defined by the sequence $\left\{p_{v}\right\}$.

It is occasionally of interest to compare the definition (1.04) with the following:

$$
\left(R, p_{\nu}\right)-\lim x_{n}=\lim _{n \rightarrow \infty} P_{n}^{-1}\left(p_{0} x_{0}+p_{1} x_{1}+\cdots+p_{n} x_{n}\right)
$$

subject to the regularity condition

$$
\sum_{k=0}^{n}\left|p_{k}\right|<C\left|P_{n}\right|, \quad\left|P_{n}\right| \rightarrow \infty,
$$

which definition played a rôle in the early development of the typical means of M. Riesz (cf. G. H. Hardy [2]).

We shall consider four different classes of trigonometric series which will be referred to by the symbols $L, \tilde{L}, L^{\prime}$ and $\tilde{L}^{\prime}$ in the following. With each such series there is associated a sum-function defined almost everywhere, e.g. the Abel-Poisson sum of the series. We use the same symbol for the class of sum functions as for the corresponding class of series. Thus $L$ denotes both the class of all measurable functions, periodic with period $2 \pi$ and integrable in the sense of Lebesgue over the interval $(-\pi, \pi)$, and the class of all FourierLebesgue series, the association being

$$
f(x) \sim \sum_{-\infty}^{\infty} f_{n} e^{n i x}, f_{n}=\frac{1}{2 \pi} \int_{-\pi}^{\pi} f(t) e^{-n i t} d t .
$$

The conjugate series of the Fourier series of $f(x)$ is

$$
-i \sum_{-\infty}^{\infty} \operatorname{sgn}(n) f_{n} e^{n i x}
$$

the corresponding sum-function being 


$$
\tilde{f}(x) \equiv-\frac{1}{2 \pi} \lim _{\rightarrow 00} \int_{\epsilon}^{\pi}[f(x+t)-f(x-t)] \cot \frac{t}{2} d t,
$$

which exists almost everywhere. The class of all such series or functions is denoted by $\tilde{L}$. If $f(x)$ is of bounded variation, the series

$$
\begin{gathered}
i \sum_{-\infty}^{+\infty} n f_{n} e^{n i x}, \\
\sum_{-\infty}^{+\infty}|n| f_{n} e^{n i x}
\end{gathered}
$$

are members of the classes $L^{\prime}$ and $\tilde{L}^{\prime}$ respectively, the corresponding sum functions being $f^{\prime}(x)$ and

(1.13) $\tilde{f}^{\prime}(x) \equiv-\frac{1}{4 \pi} \lim _{t \rightarrow 0} \int_{t}^{\pi}[f(x+t)+f(x-t)-2 f(x)]\left[\sin \frac{t}{2}\right]^{-2} d t$ respectively.

2. The effectiveness problem. Now let $\mathfrak{T}$ be a class of trigonometric series

$$
\sum_{-\infty}^{+\infty} F_{n} e^{n i x},
$$

each series having an associated generalized sum-function $F(x)$. In the sequel $\mathfrak{I}$ will be one of the four classes defined above, but the following considerations have a sense in more general cases. Put

and substitute

in (1.01) so that

$$
F_{n}(x)=\sum_{k=-n}^{+n} F_{k} e^{k i x}
$$

$$
x_{n}=F_{n}(x), y_{m}=\tau_{m}(x, F)
$$

$$
\tau_{m}(x, F)=\sum_{k=-m}^{+m}\left\{\sum_{n=|k|}^{m} a_{m n}\right\} F_{k} e^{k i x} .
$$

Let $E_{F}$ be a point set in the interval $(-\pi, \pi)$ defined for each function $F(x) \subset \mathfrak{I}$ and such that, at every point $x \subset E_{F}, F(x)$ has a finite definite value and satisfies a prescribed condition of regularity. $E_{F}$ will usually vary from one function to the other in $\mathfrak{T}$ and may be vacuous.

Definition 1. The method of summation $A$ is said to be $\left(\mathfrak{I}, E_{F}\right)$-effective if

$$
\lim _{m \rightarrow \infty} \tau_{m}(x, F)=F(x)
$$

whenever $F(x) \subset \mathfrak{T}$ and $x \subset E_{F}$. 
We are not aware of any general attack on this $\left(\mathfrak{T}, E_{F}\right)$-effectiveness problem, and we are not able to solve it in its generality. But in view of the importance of this range of questions we consider it worth while to solve the problem for subclasses of $\mathcal{A}$ and for particular choices of $\mathfrak{T}$ and $E_{F}$. Thus in the present paper we are concerned with the class $\mathcal{N}$, and in order to get satisfactory results we have to impose fairly severe restrictions on the generating sequence $\left\{p_{n}\right\}$ some of which are undoubtedly due to imperfections of the method.

The class $\mathfrak{T}$ will be any one of the four classes $L, \tilde{L}, L^{\prime}$ and $\tilde{L}^{\prime}$ mentioned above. We consider six different types of sets $E_{F}$, viz. two in connection with each of the classes $L$ and $\tilde{L}$ and one for each of the classes $L^{\prime}$ and $\tilde{L}^{\prime}$. These are the sets of $\left(^{*}\right)$-regular points where the asterisk represents one of the six symbols $F, L, \tilde{F}, \tilde{L}, L^{\prime}$ and $\tilde{L}^{\prime}$. These sets will now be defined. In the following $f(x) \subset L$ and the set of values of $x$ for which $f(x)$ does not have a finite definite value is disregarded. The following notation will be employed:

$$
\begin{aligned}
f(x+t)+f(x-t)-2 f(x) & \equiv \phi(t), \\
\int_{0}^{t} \phi(s) d s & \equiv \phi_{1}(t), \\
\int_{0}^{t}|\phi(s)| d s & \equiv \Phi(t), \\
f(x+t)-f(x-t) & \equiv \psi(t), \\
\int_{0}^{t} \psi(s) d s & \equiv \psi_{1}(t), \\
\int_{0}^{t}|\psi(s)| d s & \equiv \Psi(t), \\
f(x+t)-f(x-)^{\prime}-2 t f^{\prime}(x) & \equiv \chi(t), \\
\int_{0}^{t}\left|d_{s} \phi(s)\right| & \equiv \phi_{0}(t), \\
\int_{0}^{t}\left|d_{s} \chi(s)\right| & \equiv \chi_{0}(t) .
\end{aligned}
$$

In the last three formulas $f(x)$ is supposed to be of bounded variation and we disregard the set of measure zero in which $f^{\prime}(x)$ does not exist as a finite number.

Defrnition 2. A point $x$ for which $f(x)$ has a finite definite value is said to be

(i) (F)-regular if $\phi(t) \rightarrow 0$ with $t$;

(ii) (L)-regular if $\Phi(t)=o(t)$; 
(iii) $(\tilde{F})$-regular if it is a point of continuity of $f(x)$ and $\tilde{f}(x)$ exists and is finite (see formula (1.10));

(iv) $(\tilde{L})$-regular if $\Psi(t)=o(t)$ and $\tilde{f}(x)$ exists and is finite;

(v) $\left(L^{\prime}\right)$-regular if $f(u)$ is of bounded variation in $(-\pi, \pi), f^{\prime}(x)$ exists and is finite and $\chi_{0}(t)=o(t)$;

(vi) (站)-regular if $f(u)$ is of bounded variation in $(-\pi, \pi), \tilde{f}^{\prime}(x)$ exists and is finite (see formula (1.13)) and $\phi_{0}(t)=o(t)$.

We designate the set of $\left(^{*}\right)$-regular points with respect to a given function $f(x)$ in the interval $(-\pi, \pi)$ by $E\left(^{*} ; f\right)$. We have clearly

$$
\begin{aligned}
& E(L ; f)>E(F ; f)>E(\tilde{F} ; f), \\
& E(\tilde{L} ; f)>E(\tilde{F} ; f) .
\end{aligned}
$$

It is well known that the sets $E(L ; f), E(\tilde{L} ; f), E\left(L^{\prime} ; f\right)$ and $E\left(\tilde{L}^{\prime} ; f\right)$ all have the measure $2 \pi$.

Definition 3. A method of summation which is (T, $\left.E_{F}\right)$-effective is said to be

(i) (F)-effective if $\mathfrak{T}=L, E_{F}=\dot{E}(F ; f)$;

(ii) (L)-effective if $\mathfrak{T}=L, E_{F}=E(L ; f)$;

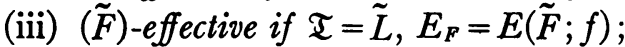

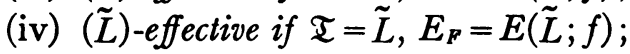

(v) $\left(L^{\prime}\right)$-effective if $\mathfrak{T}=L^{\prime}, E_{F}=E\left(L^{\prime} ; f\right)$;

(vi) $\left(\tilde{L}^{\prime}\right)$-effective if $\mathfrak{T}=\tilde{L}^{\prime}, E_{F}=E\left(\tilde{L}^{\prime} ; f\right)$;

(vii) Fourier-effective if it is effective in the sense of (i)-(vi) simultaneously.

The obvious implications between the different types of effectiveness are

$$
\begin{aligned}
& (L) \rightarrow(F), \\
& (\tilde{L}) \rightarrow(\tilde{F}) .
\end{aligned}
$$

3. Summary of the results. The main result of our investigation is

TheOREM I. A regular Nörlund method of summation $\left(N, p_{v}\right)$ is Fouriereffective if the generating sequence $\left\{p_{v}\right\}$ satisfies the following conditions:

$$
\begin{aligned}
n\left|p_{n}\right| & <C\left|P_{n}\right|, \\
\sum_{k=1}^{n} k\left|p_{k}-p_{k-1}\right| & <C\left|P_{n}\right|, \\
\sum_{k=1}^{n} \frac{\left|P_{k}\right|}{k} & <C\left|P_{n}\right|,
\end{aligned}
$$

where $C$ is a fixed constant independent of $n$. 
These conditions are certainly not necessary in general, but they are partly necessary in what is perhaps the most important case, as is shown by

TheOREM II. If $p_{n}>0$ and $\left\{p_{v}\right\}$ satisfies (3.01) and (3.02), then condition (3.03) becomes necessary as well as sufficient for the regular method $\left(N, p_{v}\right)$ to be $(F)$-effective.

The proofs of these theorems occupy $\$ \S 4-8$ and $\$ 9$ respectively. $\$ 9$ also contains a more detailed study of the method $\left(N, p_{v}\right)$ in the case where $p_{v}>0$ and $p_{v}$ is ultimately monotone, or in the more general case where conditions (3.01), (3.02) are satisfied (local sufficient conditions for summability when (3.03) does not hold and determination of the order of the Lebesgue constants together with construction of methods with given order of growth of the Lebesgue constants). $\$ 10$ contains special examples. $\$ 11$ contains some results based on the fact that any definition of summability which includes $(C, 1)$ (in the terminology of W. A. Hurwitz) is also Fourier-effective.

4. Basic formulas. We assume $f(u) \subset L$,

$$
f(u) \sim \sum_{-\infty}^{\infty} f_{k} e^{k i u}
$$

and put

$$
\begin{gathered}
s_{n}(x)=\sum_{-n}^{n} f_{k} e^{k i x}, \\
\tilde{s}_{n}(x)=-i \sum_{-n}^{n} \operatorname{sgn}(k) f_{k} e^{k i x}, \\
\sigma_{n}(x)=s_{n}(x)+i \tilde{s_{n}}(x)=f_{0}+2 \sum_{1}^{n} f_{k} e^{k i x} .
\end{gathered}
$$

We form the $n$th $\left(N, p_{v}\right)$-mean of the sequence $\left\{F_{m}(x)\right\}$

$$
\tau_{n}(x, F)=\frac{1}{P_{n}} \sum_{k=0}^{n} p_{n-k} F_{k}(x),
$$

substituting for $F_{k}(x)$ successively $s_{k}(x), \tilde{s}_{k}(x)$ and $\sigma_{k}(x)$, and we denote the results by $N_{n}\left[f(x), p_{\nu}\right], \tilde{N}_{n}\left[f(x), p_{\nu}\right]$ and $\mathfrak{N}_{n}\left[f(x), p_{\nu}\right]$ respectively. We write

$$
\begin{aligned}
\mathfrak{R}_{n}\left[f(x), p_{\nu}\right] & =\int_{-\pi}^{\pi} f(x+t) \mathfrak{R}_{n}(t) d t \\
& =\int_{-\pi}^{\pi} f(x+t)\left[N_{n}(t)+i \tilde{N}_{n}(t)\right] d t \\
& =N_{n}\left[f(x), p_{\nu}\right]+i \tilde{N}_{n}\left[f(x), p_{\nu}\right] .
\end{aligned}
$$




\section{Putting}

(4.06) $\mathfrak{P}_{n}(t)=\sum_{k=0}^{n} p_{k} e^{k i t}=\sum_{k=0}^{n} p_{k} \cos k t+i \sum_{k=0}^{n} p_{k} \sin k t=\mathfrak{C}_{n}(t)+i \Im_{n}(t)$,

we have

$$
\begin{aligned}
\Re_{n}(t) & =N_{n}(t)+i \tilde{N}_{n}(t)=\frac{1}{2 \pi P_{n}} \sum_{k=0}^{n} p_{n-k}\left[1+2 \sum_{m=1}^{k} e^{-m i t}\right] \\
& =\frac{i}{2 \pi}\left[\left(P_{n} \sin \frac{t}{2}\right)^{-1} e^{-(n+1 / 2) i t} \mathfrak{P}_{n}(t)-\cot \frac{t}{2}\right]
\end{aligned}
$$

and

$$
\begin{aligned}
N_{n}(t) & =i\left\{4 \pi P_{n} \sin \frac{t}{2}\right\}^{-1}\left\{e^{-(n+1 / 2) i t} \mathfrak{B}_{n}(t)-e^{(n+1 / 2) i t} \mathfrak{B}_{n}(-t)\right\} \\
& =\left\{2 \pi P_{n} \sin \frac{t}{2}\right\}^{-1}\left\{\mathfrak{\complement}_{n}(t) \sin \left(n+\frac{1}{2}\right) t-\mathfrak{S}_{n}(t) \cos \left(n+\frac{1}{2}\right) t\right\} .
\end{aligned}
$$

We note also that

$$
N_{n}(t)=\frac{1}{2 \pi P_{n}} \sum_{k=0}^{n} p_{n-k} \frac{\sin \left(k+\frac{1}{2}\right) t}{\sin \frac{1}{2} t}
$$

which shows that

$$
\int_{-\pi}^{\pi} N_{n}(t) d t=1
$$

Assuming $f(u)$ to have a finite definite value for $u=x$, and using the notation of (2.05) together with the fact that $N_{n}(t)$ is an even function, we get

$$
N_{n}\left[f(x), p_{\nu}\right]-f(x)=\int_{0}^{\pi} \phi(t) N_{n}(t) d t
$$

The conjugate function $\tilde{f}(x)$ is defined for almost every $x$, in particular for $x \subset E(\tilde{L} ; f)$, by

$$
\tilde{f}(x)=-\frac{1}{2 \pi} \lim _{a \rightarrow 0} \int_{\epsilon}^{\pi} \psi(t) \cot \frac{t}{2} d t
$$

(see (1.10) and (2.08)). It follows that for $x \subset E(\tilde{L} ; f)$

$$
\tilde{N}_{n}\left[f(x), p_{\nu}\right]-\tilde{f}(x)=\lim _{\epsilon \rightarrow 0} \int_{0}^{\pi} \psi(t) \bar{N}_{n}(t) d t,
$$

where 


$$
\begin{aligned}
\bar{N}_{n}(t) & \equiv \tilde{N}_{n}(t)+\frac{1}{2 \pi} \cot \frac{t}{2} \\
& =\left\{4 \pi P_{n} \sin \frac{t}{2}\right\}^{-1}\left\{e^{-(n+1 / 2) i t} \mathfrak{B}_{n}(t)+e^{(n+1 / 2) i t} \mathfrak{B}_{n}(-t)\right\} \\
& =\left\{2 \pi P_{n} \sin \frac{t}{2}\right\}^{-1}\left\{\Im_{n}(t) \sin \left(n+\frac{1}{2}\right) t+\left(\Im_{n}(t) \cos \left(n+\frac{1}{2}\right) t\right\}\right.
\end{aligned}
$$

We now pass over to the derived series and to its conjugate series, i.e. the series

$$
\begin{gathered}
i \sum_{-\infty}^{+\infty} k f_{k} e^{k i x}, \\
\sum_{-\infty}^{+\infty}|k| f_{k} e^{k i x},
\end{gathered}
$$

assuming $f(u)$ to be of bounded variation in $(-\pi, \pi)$. The $n$th partial sums of these two series are $s_{n}^{\prime}(x)$ and $\tilde{s}_{n}^{\prime}(x)$ respectively (see (4.02) and (4.03)). The $n$th $\left(N, p_{v}\right)$-mean of the sequence $\left\{\sigma_{m}^{\prime}(x)\right\}$ is

$$
\mathfrak{N}_{n}^{\prime}\left[f(x), p_{\nu}\right]=\int_{-\pi}^{\pi} \mathfrak{N}_{n}(t) d_{t} f(x+t)=N_{n}^{\prime}\left[f(x), p_{\nu}\right]+i \tilde{N}_{n}^{\prime}\left[f(x), p_{\nu}\right],
$$

where the prime denotes differentiation with respect to $x$ and the symbols have the sense defined above. Assuming $f^{\prime}(x)$ to exist, we get

$$
N_{n}^{\prime}\left[f(x), p_{\nu}\right]-f^{\prime}(x)=\int_{0}^{\pi} N_{n}(t) d \chi(t) .
$$

Further,

But

$$
\begin{aligned}
\tilde{N}_{n}^{\prime}\left[f(x), p_{\nu}\right] & =\int_{0}^{\pi} \tilde{N}(t) d \phi(t) \\
& =\int_{0}^{1 / n} \tilde{N}_{n}(t) d \phi(t)+\int_{1 / n}^{\pi} \bar{N}_{n}(t) d \phi(t)-\frac{1}{2 \pi} \int_{1 / n}^{\pi} \cot \frac{-}{2} d \phi(t) .
\end{aligned}
$$

$$
-\frac{1}{2 \pi} \int_{\epsilon}^{\pi} \cot \frac{t}{2} d \phi(t)=\frac{1}{2 \pi} \cot \frac{\epsilon}{2} \phi(\epsilon)-\frac{1}{4 \pi} \int_{\epsilon}^{\pi} \phi(t)\left[\sin \frac{t}{2}\right]^{-2} d t .
$$

At an $\left(\tilde{L}^{\prime}\right)$-regular point $\phi(\epsilon)=o(\epsilon)$ since this estimate holds for $\phi_{0}(\epsilon)$. It follows that the first term on the right tends to zero and the second one tends to $\tilde{f}^{\prime}(x)$ as $\epsilon \rightarrow 0$. Hence

(4.19) $\tilde{N}_{n}^{\prime}\left[f(x), p_{\nu}\right]-\tilde{f}^{\prime}(x)=\int_{0}^{1 / n} \tilde{N}_{n}(t) d \phi(t)+\int_{1 / n}^{\pi} \bar{N}_{n}(t) d \phi(t)+o(1)$ at $\left(\tilde{L}^{\prime}\right)$-regular points. 
5. Preliminary lemmas. The subsequent discussion will center around the singular integrals in formulas (4.11), (4.13), (4.18) and (4.19). It will be as= sumed throughout that the sequence $\left\{p_{\nu}\right\}$ satisfies conditions (1.05) so that $\left(N, p_{v}\right)$ is a regular definition of summation.

Formula (4.11) offers the simplest problem especially when $x \subset E(F ; f)$. Necessary conditions in order that the integral in (4.11) shall tend to zero for every $f(u) \subset L$ at all points $x \subset E(F ; f)$ are well known. One condition is that

$$
\int_{\alpha}^{\beta} N_{n}(t) d t \rightarrow 0
$$

as $n \rightarrow \infty,(\alpha, \beta)$ being any closed sub-interval of $(0, \pi)$ not containing the origin. This condition is satisfied by virtue of regularity conditions (1.05), and (4.09), since

$$
\lim _{n \rightarrow \infty} \int_{\alpha}^{\beta} \frac{\sin \left(n+\frac{1}{2}\right) t}{\sin (t / 2)} d t=0
$$

for any fixed $\alpha, \beta$ provided $0<\alpha<\beta \leqq \pi$.

The second, and more restrictive, condition is that

$$
\int_{0}^{\pi}\left|N_{n}(t)\right| d t<C
$$

for some finite $C$ independent of $n$. This condition is not satisfied merely by virtue of regularity conditions, and the crux of the problem is the investigation of (5.02).

In the discussion of this and analogous integrals we can disregard the intervals $(0,1 / n)$ and $(\delta, \pi)$ for a fixed positive $\delta$ if $x$ and $\left\{p_{\nu}\right\}$ respectively are suitably restricted. This follows from the following lemmas.

LEMMA 1. We have

$$
\mathfrak{N}_{n}(t)=O(n) .
$$

The same estimate holds for $N_{n}(t)$ and $\tilde{N}_{n}(t)$. Further

By definition

$$
\bar{N}_{n}(t)=\frac{1}{2 \pi} \cot \frac{t}{2}+O(n) .
$$

so that

$$
\mathfrak{N}_{n}(t)=\frac{1}{2 \pi P_{n}} \sum_{k=0}^{n} p_{n-k}\left[1+2 \sum_{m=1}^{k} e^{-m i t}\right]
$$

$$
\left|\mathfrak{N}_{n}(t)\right| \leqq \frac{1}{2 \pi\left|P_{n}\right|} \sum_{k=0}^{n}(2 k+1)\left|p_{n-k}\right| \leqq \frac{2 n+1}{2 \pi\left|P_{n}\right|} \sum_{k=0}^{n}\left|p_{n-k}\right| .
$$


This estimate together with (1.05) shows that (5.03) holds. The estimates for the other functions follow from their definitions together with (5.03).

LEMMA 2. If $x \subset E(L ; f)$, then

$$
\int_{0}^{1 / n} \phi(t) N_{n}(t) d t=o(1) .
$$

If $f(u)$ is of bounded variation in $(-\pi, \pi)$ and $x \subset E\left(L^{\prime} ; f\right)$, then

$$
\int_{0}^{1 / n} N_{n}(t) d \chi(t)=o(1) \text {. }
$$

In view of Definition 2, Lemma 1, and formulas (2.07), (2.11) and (2.13)

$$
\begin{aligned}
& \int_{0}^{1 / n} \phi(t) N_{n}(t) d t=O\left\{n \Phi\left(\frac{1}{n}\right)\right\}=o(1), \\
& \int_{0}^{1 / n} N_{n}(t) d \chi(t)=O\left\{n \chi_{0}\left(\frac{1}{n}\right)\right\}=o(1) .
\end{aligned}
$$

LEMMA 3. If $x \subset E(\tilde{L} ; f)$, then

$$
\lim _{\epsilon \rightarrow 0} \int_{e}^{1 / n} \bar{N}_{n}(t) \psi(t) d t=o(1) .
$$

We have

$$
\lim _{\epsilon \rightarrow 0} \int_{\epsilon}^{1 / n} \psi(t) \bar{N}_{n}(t) d t=\lim _{\epsilon \rightarrow 0} \int_{\epsilon}^{1 / n} \psi(t) \tilde{N}_{n}(t) d t+\frac{1}{2 \pi} \lim _{\epsilon \rightarrow 0} \int_{\epsilon}^{1 / n} \psi(t) \cot \frac{t}{2} d t .
$$

Here the first expression on the right is

$$
O\left\{n \Psi\left(\frac{1}{n}\right)\right\}=o(1)
$$

by Definition 2 (iv), Lemma 1 , and formulas (2.08) and (2.10), whereas the second expression is $o(1)$ since $\tilde{f}(x)$ exists (cf. formula (4.12)).

LEMma 4. If $x \subset E\left(\tilde{L}^{\prime} ; f\right)$, then

$$
\int_{0}^{1 / n} \tilde{N}_{n}(t) d \phi(t)=o(1) .
$$

By Definition 2 and Lemma 1,

$$
\left|\int_{0}^{1 / n} \tilde{N}_{n}(t) d \phi(t)\right|=O\left\{n \phi_{(}\left(\frac{1}{n}\right)\right\}=o(1) .
$$


Lемма 5. If $\left\{p_{\nu}\right\}$ satisfies the condition

$$
\sum_{k=1}^{n}\left|p_{k}-p_{k-1}\right|=o\left(P_{n}\right)
$$

then

$$
\mathfrak{P}_{n}(t)=o\left(P_{n}\right)
$$

uniformly in $t$ for $0<\delta \leqq|t| \leqq \pi$.

We have

$$
\mathfrak{B}_{n}(t)=\sum_{k=0}^{n} p_{k} e^{k i t}=\sum_{k=0}^{n-1}\left(p_{k}-p_{k+1}\right) S_{k}+p_{n} S_{n}
$$

where

$$
S_{k}=\frac{e^{(k+1) i t}-1}{e^{i t}-1} .
$$

Hence by (1.05) and (5.09)

$$
\left|\mathfrak{P}_{n}(t)\right| \leqq\left\{\sum_{0}^{n-1}\left|p_{k}-p_{k+1}\right|+\left|p_{n}\right|\right\} O\left(\frac{1}{t}\right)=o\left(P_{n}\right) \cdot O\left(\frac{1}{t}\right)
$$

where the " $o$ " refers to $n \rightarrow \infty$ and is independent of $t$, and the " $O$ " refers to $t \rightarrow 0$ and is independent of $n$. This proves the lemma.

Condition (5.09) certainly holds if conditions (3.02) and (3.03) of Theorem I are satisfied. Indeed, then $\left|P_{n}\right| \rightarrow \infty$, and if

then

$$
W_{n} \equiv \sum_{k=1}^{n} k\left|p_{k}-p_{k-1}\right|=O\left(P_{n}\right), W_{0} \equiv 0,
$$

$$
V_{n} \equiv \sum_{k=1}^{n}\left|p_{k}-p_{k-1}\right|=\sum_{k=1}^{n} \frac{1}{k}\left(W_{k}-W_{k-1}\right)=\sum_{k=1}^{n-1} \frac{W_{k}}{k(k+1)}+\frac{W_{n}}{n}
$$

from which the conclusion follows immediately. The conclusion holds if only (3.02) is satisfied and $\left|P_{n}\right| \rightarrow \infty$. Otherwise (still under assumption of (3.02)) it is readily found that the series $\sum_{1}^{\infty} k\left|p_{k}-p_{k-1}\right|$ converges, and

$$
\sum_{k=m}^{m+p}\left|p_{k}-p_{k-1}\right|=o\left(\frac{1}{m}\right) \text {. }
$$

LEMMA 6. If $\left\{p_{\nu}\right\}$ satisfies condition (5.09), then the integrals

$$
\int_{\delta}^{\pi} N_{n}(t) \phi(t) d t, \quad \int_{\delta}^{\pi} \bar{N}_{n}(t) \psi(t) d t, \quad \int_{\delta}^{\pi} N_{n}(t) d \chi(t), \int_{\delta}^{\pi} \bar{N}_{n}(t) d \phi(t)
$$

tend to zero as $n \rightarrow \infty, \delta$ being fixed $>0$. 
We have merely to show that the kernels tend to zero uniformly in $t$, $0<\delta \leqq t \leqq \pi$, as $n \rightarrow \infty$. But this follows from Lemma 5 together with formulas (4.07) and (4.14).

The discussion is consequently reduced to the interval $(1 / n, \delta)$.

6. Estimates of the kernels. We have to find suitable estimates for the kernels $N_{n}(t)$ and $\bar{N}_{n}(t)$ in the interval $(1 / n, \delta)$. Such estimates can be found by fairly crude methods which nevertheless give rather accurate results provided the sequence $\left\{p_{v}\right\}$ is sufficiently regular. Since $\mathfrak{P}_{n}(t)$ enters in both kernels we start by estimating this function.

We put

$$
\left|p_{n}\right|=r_{n}, \quad R_{n}=r_{0}+r_{1}+\cdots+r_{n},
$$

and introduce the step functions

$$
r(u)=r_{[u]}, R(u)=R_{[u]}
$$

where $[u]$ as usual denotes the largest integer $\leqq u$. Finally we put

$$
V_{0} \equiv 0, \quad V_{n} \equiv \sum_{k=1}^{n}\left|p_{k}-p_{k-1}\right|, \quad V(u) \equiv V_{[u]}
$$

\section{Consider}

$$
\mathfrak{P}_{n}(t)=\sum_{k=0}^{n} p_{k} e^{k i t}=\Sigma_{1}+\Sigma_{2} \quad(t>0)
$$

where $k$ ranges over the integers $\leqq \tau=[1 / t]$ in $\Sigma_{1}$ and over the integers $>\tau$ but $\leqq n$ in $\Sigma_{2}$. It is clear that

$$
\left|\Sigma_{1}\right| \leqq \sum_{k=0}^{\tau}\left|p_{k}\right|=\sum_{k=0}^{\tau} r_{k}=R_{\tau}=R\left(\frac{1}{t}\right) .
$$

Further,

$$
\Sigma_{2}=-p_{\tau} S_{\tau}+\sum_{k=\tau}^{n-1}\left(p_{k}-p_{k+1}\right) S_{k}+p_{n} S_{n}
$$

where $S_{k}$ is defined by (5.11). Since $\left|t S_{k}\right| \leqq \pi$, we get

$$
\left|\Sigma_{2}\right| \leqq \frac{\pi}{t}\left\{\left|p_{\tau}\right|+\sum_{k=\tau}^{n-1}\left|p_{k}-p_{k+1}\right|+\left|p_{n}\right|\right\}
$$

or

$$
\left|\Sigma_{2}\right| \leqq \frac{\pi}{t}\left\{r\left(\frac{1}{t}\right)+r(n)+V(n)-V\left(\frac{1}{t}-1\right)\right\} .
$$


The same estimates apply for $t<0$. Consequently

$$
\left|\mathfrak{P}_{n}( \pm t)\right| \leqq R\left(\frac{1}{t}\right)+\frac{\pi}{t}\left\{r\left(\frac{1}{t}\right)+r(n)+V(n)-V\left(\frac{1}{t}-1\right)\right\}
$$

and referring to formulas (4.08) and (4.14) we see that

$$
\left.\mid \begin{array}{l}
N_{n}(t) \mid \\
\left|\bar{N}_{n}(t)\right|
\end{array}\right\} \leqq
$$

$$
\frac{1}{2 t\left|P_{n}\right|}\left\{R\left(\frac{1}{t}\right)+\frac{\pi}{t}\left[r\left(\frac{1}{t}\right)+r(n)+V(n)-V\left(\frac{1}{t}-1\right)\right]\right\} .
$$

By virtue of (1.05) this estimate implies the existence of a positive constant $A$ such that

$$
\left.\mid \begin{array}{l}
\left|N_{n}(t)\right| \\
\left|\bar{N}_{n}(t)\right|
\end{array}\right\} \leqq A\left\{M_{n 1}(t)+M_{n 2}(t)+M_{n 3}(t)+M_{n 4}(t)\right\}
$$

where

$$
\begin{array}{ll}
M_{n 1}(t)=\frac{1}{t R(n)} R\left(\frac{1}{t}\right), & M_{n 2}(t)=\frac{1}{t^{2} R(n)} r\left(\frac{1}{t}\right) \\
M_{n 3}(t)=\frac{r(n)}{t^{2} R(n)}, & M_{n 4}(t)=\frac{1}{t^{2} R(n)}\left\{V(n)-V\left(\frac{1}{t}-1\right)\right\} .
\end{array}
$$

7. The Fejér cases. In order that

$$
\int_{1 / n}^{\delta} \phi(t) N_{n}(t) d t=o(1)
$$

at $(F)$-regular points it is sufficient that

$$
\int_{1 / n}^{\delta}\left|N_{n}(t)\right| d t<C
$$

and in order that

$$
\int_{1 / n}^{\delta} \psi(t) \bar{N}_{n}(t) d t=o(1)
$$

at $(\tilde{F})$-regular points it is sufficient that

$$
\int_{1 / n}^{\delta}\left|\bar{N}_{n}(t)\right| d t<C
$$

That conditions (7.02) and (7.04) are satisfied under the assumptions of Theorem I follows from 
LEMMA 7. If the sequence $\left\{p_{p}\right\}$ satisfies conditions (3.01), (3.02) and (3.03), then

$$
\int_{1 / n}^{\delta} M_{n m}(t) d t \leqq M \quad(m=1,2,3,4) .
$$

Property (7.05) in the cases $m=2,3,4$ holds if we assume only that conditions (3.01), (3.02) are satisfied.

For $m=1$ we have

$$
\int_{1 / n}^{\delta} M_{n 1}(t) d t=\frac{1}{R(n)} \int_{1 / n}^{\delta} R\left(\frac{1}{t}\right) \frac{d t}{t}=\frac{1}{R(n)} \int_{1 / 8}^{n} R(s) \frac{d s}{s}
$$

which is bounded if and only if

$$
\frac{1}{R_{n}} \sum_{k=1}^{n} \frac{R_{k}}{k}
$$

is bounded, and this is implied by and implies condition (3.03).

For $m=2$ we have simply

$$
\int_{1 / n}^{\delta} M_{n 2}(t) d t=\frac{1}{R(n)} \int_{1 / n}^{\delta} r\left(\frac{1}{t}\right) \frac{d t}{t^{2}}=\frac{1}{R(n)} \int_{1 / \delta}^{n} r(s) d s<1 .
$$

For $m=3$

$$
\int_{1 / n}^{\delta} M_{n 8}(t) d t=\frac{r(n)}{R(n)} \int_{1 / n}^{\delta} \frac{d t}{t^{2}}<\frac{n r_{n}}{R_{n}}
$$

which is bounded by virtue of (3.01).

Finally for $m=4$

$$
\begin{aligned}
\int_{1 / n}^{\delta} M_{n 4}(t) d t & =\frac{1}{R(n)} \int_{1 / n}^{\delta}\left[V(n)-V\left(\frac{1}{t}-1\right)\right] \frac{d t}{t^{2}} \\
& =\frac{1}{R(n)} \int_{1 / 8}^{n}[V(n)-V(s-1)] d s<\frac{1}{R(n)} \int_{0}^{n}[V(n)-V(s)] d s \\
& =\frac{1}{R(n)} \int_{0}^{n} s d V(s)=\frac{1}{R_{n}} \sum_{k=1}^{n} k\left|p_{k}-p_{k-1}\right|
\end{aligned}
$$

which is bounded by (3.02).

We have consequently proved that part of Theorem I which refers to $(F)$ - and $(\tilde{F})$-effectiveness. 
8. The Lebesgue cases. The problem which confronts us in the four Lebesgue cases, $(L),(\tilde{L}),\left(L^{\prime}\right)$ and $\left(\tilde{L}^{\prime}\right)$, is of the following form: $\omega(t)$ is a function of bounded variation such that

$$
\omega_{0}(t) \equiv \int_{0}^{t}|d \omega(s)|=o(t) ;
$$

find sufficient conditions in order that

$$
\int_{1 / n}^{\delta} K_{n}(t) d \omega(t)=o(1) .^{*}
$$

In the four cases the symbols have the following meaning:

\begin{tabular}{|c|c|c|}
\hline Case & $K_{n}(t)$ & $\omega(t)$ \\
\hline$(L)$ & $N_{n}(t)$ & $\phi_{1}(t)$ \\
\hline$(\tilde{L})$ & $\bar{N}_{n}(t)$ & $\psi_{1}(t)$ \\
\hline$\left(L^{\prime}\right)$ & $N_{n}(t)$ & $\chi(t)$ \\
\hline$\left(\tilde{L}^{\prime}\right)$ & $\bar{N}_{n}(t)$ & $\phi(t)$ \\
\hline
\end{tabular}

In order to prove the remaining part of Theorem I it is consequently sufficient to prove

Lemma 8. If $\left\{p_{\nu}\right\}$ satisfies conditions (3.01), (3.02), (3.03) of Theorem I, and if $\omega(t)$ satisfies $(8.01)$, then $\dagger$

$$
\int_{1 / n}^{\delta} M_{n m}(t) d \omega_{0}(t)=o(1) \quad(m=1,2,3,4) .
$$

Property (8.03) in the cases $m=2,3,4$ holds if we assume only that conditions (3.01), (3.02) are satisfied.

We have

$$
\begin{aligned}
& \text { (8.04) } \int_{1 / n}^{\delta} M_{n 1}(t) d \omega_{0}(t)=\frac{1}{R(n)} \int_{1 / n}^{\delta} R\left(\frac{1}{t}\right) \frac{1}{t} d \omega_{0}(t) \\
& =\frac{1}{R(n)}\left\{\left[R\left(\frac{1}{t}\right) \frac{1}{t} \omega_{0}(t)\right]_{1 / n}^{\delta}+\int_{1 / n}^{\delta} \omega_{0}(t) \frac{1}{t^{2}} R\left(\frac{1}{t}\right) d t-\int_{1 / n}^{\delta} \omega_{0}(t) \frac{1}{t} d R\left(\frac{1}{t}\right)\right\} .
\end{aligned}
$$

* By this notation we mean to say that, being given an arbitrarily small $\epsilon>0$, we can find a $\delta \equiv \delta(\epsilon)$ and an $n_{0} \equiv n_{0}(\epsilon)$ such that the left-hand member of (8.02) will be $\leqq \epsilon$ in absolute value for $n \geqq n_{0}$.

$\dagger$ The integrals here are taken in the sense of Stieltjes-Young while the interval of integration is always assumed to be closed. For the justification of the integrations by parts extensively used in the subsequent discussion we refer to the recent paper by Evans [1], Lemma II, p. 217. 
Here the integrated part is $o(1)$ since (8.01) holds and $R(n) \rightarrow \infty$, by (3.03). The second term is

$$
o\left\{\frac{1}{R(n)} \int_{1 / n}^{\delta} R\left(\frac{1}{t}\right) \frac{d t}{t}\right\}=o\left\{\frac{1}{R(n)} \int_{1}^{n} \frac{R(s)}{s} d s\right\}=o(1)
$$

by (3.03). The third term is

$$
o\left\{\frac{1}{R(n)} \int_{1 / n}^{\delta}\left|d R\left(\frac{1}{t}\right)\right|\right\}=o\left\{\frac{1}{R(n)} \int_{0}^{n} d R(s)\right\}=o(1) .
$$

This séttles the case $m=1$. Next we have

$$
\begin{aligned}
\int_{1 / n}^{\delta} M_{n 2}(t) d \omega_{0}(t)= & \frac{1}{R(n)} \int_{1 / n}^{\delta} r\left(\frac{1}{t}\right) \frac{1}{t^{2}} d \omega_{0}(t) \\
= & \frac{1}{R(n)}\left\{\left[r\left(\frac{1}{t}\right) \frac{1}{t^{2}} \omega_{0}(t)\right]_{1 / n}^{\delta}+2 \int_{1 / n}^{\delta} r\left(\frac{1}{t}\right) \omega_{0}(t) \frac{d t}{t^{3}}\right. \\
& \left.-\int_{1 / n}^{\delta} \omega_{0}(t) \frac{1}{t^{2}} d r\left(\frac{1}{t}\right)\right\}
\end{aligned}
$$

Here the integrated part is $o(1)$ by condition (3.01). The second term is

$$
o\left\{\frac{1}{R(n)} \int_{1 / n}^{\delta} r\left(\frac{1}{t}\right) \frac{d t}{t^{2}}\right\}=o\left\{\frac{\cdot 1}{R(n)} \int_{0}^{n} r(s) d s\right\}=o(1) .
$$

The third term in (8.05) is

$$
\begin{aligned}
o\left\{\frac{1}{R(n)} \int_{1 / n}^{\delta} \frac{1}{t}\left|d r\left(\frac{1}{t}\right)\right|\right\} & =o\left\{\frac{1}{R(n)} \int_{1}^{n} s|d r(s)|\right\} \\
& =o\left\{\frac{1}{R(n)} \sum_{k=1}^{n} k\left|r_{k}-r_{k-1}\right|\right\}=o(1)
\end{aligned}
$$

the sum being $O\left(R_{n}\right)$ by assumption (3.02). Hence (8.03) holds for $m=2$.

For $m=3$

$$
\int_{1 / n}^{\delta} M_{n 3}(t) d \omega_{0}(t)=\frac{r(n)}{R(n)} \int_{1 / n}^{\delta} \frac{1}{t^{2}} d \omega_{0}(t)
$$

$$
=\frac{r(n)}{R(n)}\left\{\left[\omega_{0}(t) t^{-2}\right]_{1 / n}^{\delta}+2 \int_{1 / n}^{\delta} \omega_{0}(t) t^{-3} d t\right\}=o\left(\frac{n r_{n}}{R_{n}}\right)=o(1)
$$

by assumption (3.01). 
Finally we have for $m=4$ :

$$
\begin{gathered}
\int_{1 / n}^{\delta} M_{n 4}(t) d \omega_{0}(t)=\frac{1}{R(n)} \int_{1 / n}^{\delta}\left[V(n)-V\left(\frac{1}{t}-1\right)\right] \frac{1}{t^{2}} d \omega_{0}(t) \\
(8.07)=\frac{1}{R(n)}\left\{\left(\left[V(n)-V\left(\frac{1}{t}-1\right)\right] \frac{1}{t^{2}} \omega_{0}(t)\right)_{1 / n}^{\delta}\right. \\
\left.+2 \int_{1 / n}^{\delta} \frac{1}{t^{3}}\left[V(n)-V\left(\frac{1}{t}-1\right)\right] \omega_{0}(t) d t-\int_{1 / n}^{\delta} \omega_{0}(t) \frac{1}{t^{2}} d V\left(\frac{1}{t}-1\right)\right\} .
\end{gathered}
$$

The integrated part is $o(1)$ by $(3.02){ }^{*}$ The second term is

$$
o\left\{\frac{1}{R(n)} \int_{1 / n}^{\delta}\left[V(n)-V\left(\frac{1}{t}-1\right)\right] \frac{d t}{t^{2}}\right\}=o(1)
$$

the same being true for the third term (cf. end of $\$ 7$ ). This completes the proof of Lemma 8 and hence also of Theorem II.

9. The semi-monotone case. The conditions of Theorem I are sufficient but certainly not necessary. In $\$ 11$ we shall encounter Fourier-effective definitions $\left(N, p_{v}\right)$ which do not satisfy conditions (3.01), (3.02). Condition (3.03) on the other hand seems to have intrinsic character. This is shown, at least in part, by Theorem II, the proof of which will be now given. Conditions (3.01), (3.02) are certainly satisfied if $p_{\nu}>0$ and the sequence $\left\{p_{\nu}\right\}$ is monotone decreasing. We designate as the semi-monotone case the one where $p_{\nu}>0$ and conditions (3.01), (3.02) hold. This is the hypothesis of Theorem II, which will be assumed now. Hence we have

$$
n p_{n}=O\left(P_{n}\right), V_{n}=O\left(P_{n}\right)
$$

whereas

$$
\varlimsup_{n \rightarrow \infty} \frac{1}{P_{n}} \sum_{k=1}^{n} \frac{P_{k}}{k}=+\infty .
$$

Under these assumptions we shall prove that

$$
\varlimsup_{n \rightarrow \infty} \int_{0}^{\pi}\left|N_{n}(t)\right| d t=+\infty
$$

which suffices to prove Theorem II. We use the notation of the preceding paragraphs except for the modification that $p(u)$ and $P(u)$ will replace $r(u)$ and $R(u)$ respectively.

\footnotetext{
* This is obvious when $R_{n} \rightarrow \infty$. Otherwise we use (5.12) to show that $V(n)-V(1 / \delta-1)=o(\delta)$ and so the conclusion still holds.
} 
In order to prove Theorem II we have to give a more careful estimate of $N_{n}(t)$. For this purpose we estimate $\mathfrak{\complement}_{n}(t)$ and $\Im_{n}(t)$ separately for $t>0$. Proceeding as in $\$ 6$, we write

$$
\mathfrak{G}_{n}(t)=\Sigma_{11}+\Sigma_{12}, \Im_{n}(t)=\Sigma_{21}+\Sigma_{22},
$$

where $0 \leqq k \leqq \tau$ in $\Sigma_{11}$ and $\Sigma_{21}$, and $\tau<k \leqq n$ in $\Sigma_{12}$ and $\Sigma_{22}$, and obtain the estimates

$$
\Sigma_{11}>P_{\tau} \cos 1>\frac{1}{2} P\left(\frac{1}{t}\right),
$$

$$
\left.\mid \begin{array}{c}
\Sigma_{12} \mid \\
\left|\Sigma_{22}\right|
\end{array}\right\} \leqq \frac{\pi}{t}\left\{p\left(\frac{1}{t}\right)+p(n)+V(n)-V\left(\frac{1}{t}-1\right)\right\} .
$$

Then

$$
\begin{aligned}
\int_{0}^{\pi}\left|N_{n}(t)\right| d t & >\int_{1 / n}^{1}\left|N_{n}(t)\right| d t \\
\geqq & \int_{1 / n}^{1} \frac{\left|\sin \left(n+\frac{1}{2}\right) t\right|}{2 \pi P_{n} \sin \frac{t}{2}} \Sigma_{11} d t-\int_{1 / n}^{1} \frac{\Sigma_{21} d t}{2 \pi P_{n} \sin \frac{t}{2}} \\
& -\int_{1 / n}^{1} \frac{\left|\Sigma_{12}\right| d t}{2 \pi P_{n} \sin \frac{t}{2}}-1 \int_{1 / n}^{1} \frac{\left|\Sigma_{22}\right| d t}{2 \pi P_{n} \sin \frac{t}{2}}
\end{aligned}
$$

Lemma 7 together with (9.06) shows that the last two integrals are bounded. Further

$$
\int_{1 / n}^{1} \frac{\Sigma_{21}}{2 \pi P_{n} \sin \frac{t}{2}} d t \leqq \frac{1}{2 P_{n}} \int_{1 / n}^{1} \Sigma_{21} \frac{d t}{t}=\frac{1}{2 P_{n}} \int_{1 / n}^{1}\left[\sum_{k=0}^{\tau} p_{k} \sin k t\right] \frac{d t}{t}
$$

$$
=\frac{1}{2 P_{n}} \sum_{k=1}^{n-1} p_{k} \int_{1 / n}^{1 / k} \frac{\sin k t}{t} d t<\frac{P_{n-1}}{2 P_{n}} \int_{0}^{\pi} \frac{\sin s}{s} d s<1 .
$$

By an analogous argument (Lemma 8 ) it is readily proved that

$$
\int_{1 / n}^{\delta} \frac{\Sigma_{21}}{2 \pi P_{n} \sin \frac{1}{2} t} \phi(t) d t=o(1)
$$

provided $x \subset E(L ; f)$. 
It remains to consider

$$
\begin{aligned}
\int_{1 / n}^{1} \frac{\left|\sin \left(n+\frac{1}{2}\right) t\right|}{\sin \frac{t}{2}} \Sigma_{11} d t & >\int_{1 / n}^{1}\left|\sin \left(n+\frac{1}{2}\right) t\right| P\left(\frac{1}{t}\right) \frac{d t}{t} \\
& >\frac{1}{2} \int_{E_{n}} P\left(\frac{1}{t}\right) \frac{d t}{t}
\end{aligned}
$$

where $E_{n}$ is the subset in $(1 / n, 1)$ where $\left|\sin \left(n+\frac{1}{2}\right) t\right| \geqq \frac{1}{2}$. This set consists of a number of intervals $I_{1}, I_{2}, \cdots, I_{m}$, where the enumeration proceeds from left to right. With the exception of the first and possibly also of the last interval they are all of the same length, $2 \Delta$ say, and are separated by intervals of length $\Delta$. Suppose now that $I_{1}$ is kept fixed but all other intervals are moved to the left until they abut upon each other. Denote the interval into which $I_{k}$ is carried by $J_{k}$. In this process $I_{k}$ is shifted a distance $\delta_{k}=(k-1) \Delta$ to the left, so that its left end point falls at $(2 k-3 / 2) \Delta$. It follows that any point in $J_{k}$ has a distance from the origin which exceeds two thirds of the distance from the origin of the corresponding point in $I_{k}$. Hence

$$
\begin{aligned}
\int_{I_{k}} P\left(\frac{1}{t}\right) \frac{d t}{t} & =\int_{J_{k}} P\left(\frac{1}{s+\delta_{k}}\right) \frac{d s}{s+\delta_{k}} \\
& =\int_{J_{k}} P\left(\frac{s}{s+\delta_{k}} \cdot \frac{1}{s}\right) \frac{s}{s+\delta_{k}} \cdot \frac{d s}{s}>\frac{2}{3} \int_{J_{k}} P\left(\frac{2}{3 s}\right) \frac{d s}{s}
\end{aligned}
$$

and

$$
\begin{aligned}
\int_{E_{n}} P\left(\frac{1}{t}\right) \frac{d t}{t} & >\frac{2}{3} \sum_{k=1}^{m} \int_{J_{k}} P\left(\frac{2}{3 s}\right) \frac{d s}{s} \\
& =\frac{2}{3} \int_{1 / n}^{2 /(3 \alpha)} P\left(\frac{2}{3 s}\right) \frac{d s}{s}=\frac{2}{3} \int_{\alpha}^{2 n / 3} P(u) \frac{d u}{u}
\end{aligned}
$$

where $\alpha$ is a quantity near to 1 whose numerical value is of no importance. We have consequently

$$
\int_{1 / n}^{1} \frac{\left|\sin \left(n+\frac{1}{2}\right) t\right|}{P_{n} \sin \frac{t}{2}} \Sigma_{11} d t>\frac{1}{3 P_{n}} \int_{\alpha}^{2 n / 3} P(u) \frac{d u}{u} .
$$

In order to complete the proof of (9.03) we have now merely to show that

$$
P\left(\frac{2 n}{3}\right)>\theta P(n)
$$


with a fixed positive $\theta$. Indeed, if this is the case the preceding discussion shows that the left-hand side of (9.07) cannot remain bounded as $n \rightarrow \infty$. But (9.10) is a special case of the following

Lemma 9. If $p_{n}>0$ and $n p_{n}<C P_{n}$ for every $n$, then

$$
0<\epsilon \leqq \frac{v}{u} \leqq \frac{1}{\epsilon}
$$

implies the existence of $a \delta=\delta(\epsilon)$ such that

$$
0<\delta \leqq \frac{P(v)}{P(u)} \leqq \frac{1}{\delta}
$$

We may suppose $1 \leqq u<v$ without loss of generality. Then if $u<w$,

$$
P(w)-P(u)=p(u+1)+\cdots+p(w)<C \sum_{[u]+1}^{[w]} \frac{P_{k}}{k}<C P(w) \log \frac{[w]}{[u]} .
$$

We can then choose a $\lambda>1$, independent of $u$, such that

$$
C \log \frac{[w]}{[u]}<\frac{1}{2} \text { for } w \leqq \lambda u
$$

and consequently

$$
P(u) \leqq P(w)<2 P(u) \text { if } u \leqq w \leqq \lambda u .
$$

Hence if $m$ is the least integer for which $v \leqq \lambda^{m} u$ we have

$$
P(v)<2^{m} P(u)
$$

which suffices to prove the lemma.

Hence (9.10) holds and we have finished the proof of Theorem II. A particularly important special case of this theorem is the following

THEOREM $\mathrm{II}_{1}$. If $p_{n}>0$ and $p_{n}$ is ultimately monotone decreasing, then condition (3.03) is necessary and sufficient for $(F)$-effectiveness of the method $\left(N, p_{v}\right)$, and if this condition holds, the method is Fourier-effective as well.

The assumptions imply that (3.01) and (3.02) hold so that Theorem II applies.

It follows from the proof of Theorem II together with Lemmas 7 and 8, and formula $\left(9.08^{\prime}\right)$ that if $p_{n}>0$ and (3.01) and (3.02) hold, then

$$
\lim _{n \rightarrow \infty} N_{n}\left[f(x), p_{0}\right]=f(x)
$$

at an $(L)$-regular point if and only if 


$$
\lim _{n \rightarrow \infty} \frac{1}{P_{n}} \int_{1 / n}^{\delta} \frac{\sin \left(n+\frac{1}{2}\right) t}{\sin \frac{1}{2} t}\left\{\sum_{k t \leqq 1} p_{k} \cos k t\right\} \phi(t) d t=0
$$

which is the case if and only if

$$
\lim _{n \rightarrow \infty} \frac{1}{P_{n}} \int_{1 / n}^{\delta} \sin n t\left\{\sum_{k t \leqq 1} p_{k} \cos k t\right\} \phi(t) \frac{d t}{t}=0 .
$$

But we can go a step further, as is shown by

THEOREM III. Let $p_{n}>0$, and suppose that (3.01) and (3.02) are satisfied Then

$$
\lim _{n \rightarrow \infty} \frac{1}{P_{n}} \int_{1 / n}^{\delta} \sin n t P\left(\frac{1}{t}\right) \phi(t) \frac{d t}{t}=0
$$

is a necessary and sufficient condition in order that (9.14) shall hold at an $(L)$ regular point.

Remark. Similar criteria are obtainable in this case for the summability of the conjugate, the derived, and the derived conjugate series.

The difference between the two expressions under the limit signs in (9.15) and (9.16) is $O\left(\delta_{n}\right)$ where

$$
\delta_{n}=\frac{1}{P_{n}} \int_{1 / n}^{\delta} D(t) t|\phi(t)| d t, \quad D(t)=\sum_{k t \leqq 1} k^{2} p_{k} .
$$

The usual integration by parts gives

$$
\begin{aligned}
\delta_{n} & =\frac{1}{P_{n}}\left\{[D(t) t \Phi(t)]_{1 / n}^{\delta}-\int_{1 / n}^{\delta} D(t) \Phi(t) d t-\int_{1 / n}^{\delta} \Phi(t) t d D(t)\right\} \\
& =o\left\{\frac{1}{P_{n}}\left(\left[t^{2} D(t)\right]_{1 / n}^{\delta}+\int_{1 / n}^{\delta} t D(t) d t-\int_{1 / n}^{\delta} t^{2} d D(t)\right)\right\} \\
& =o\left\{\frac{1}{P_{n}}\left(\left[t^{2} D(t)\right]_{1 / n}^{\delta}-\int_{1 / n}^{\delta} t^{2} d D(t)\right)\right\}
\end{aligned}
$$

where the last step follows from a second integration by parts. But

and

$$
t^{2} D(t)=O\left\{P\left(\frac{1}{t}\right)\right\}
$$

$$
0<-\int_{1 / n}^{\delta} t^{2} d D(t) \leqq \sum_{k=1}^{n} k^{-2} \cdot k^{2} p_{k}<P_{n}
$$

which shows that $\delta_{n}=o(1)$, and proves the theorem. 
The following sufficient condition for $\left(N, p_{v}\right)$-summability is analogous to a well known convergence criterion due to Lebesgue.

THEOREM IV. If $p_{n}>0$ and if $\left\{p_{p}\right\}$ satisfies (3.01) and (3.02), and if, for a particular $x \subset E(L ; f)$,

$$
\lim _{n \rightarrow \infty} \frac{1}{P_{n}} \int_{\pi / n}^{\delta}\left|\phi\left(t+\frac{\pi}{n}\right)-\phi(t)\right| P\left(\frac{1}{t}\right) \frac{d t}{t}=0,
$$

then (9.14) holds.

Formula (9.16) is our point of departure. A glance at the proof of Lemma 1 shows that we can replace $1 / n$ by $\pi / n \equiv \eta$ in the lower limit of this integral. Now

$$
\begin{aligned}
2 \int_{0}^{\delta} & (\sin n t) P\left(\frac{1}{t}\right) \phi(t) \frac{d t}{t} \\
= & \int_{\eta}^{\delta-\eta}(\sin n s)[\phi(s)-\phi(s+\eta)] P\left(\frac{1}{s}\right) \frac{d s}{s} \\
& +\int_{\eta}^{\delta-\eta}(\sin n s)\left[P\left(\frac{1}{s}\right)-P\left(\frac{1}{s+\eta}\right)\right] \phi(s+\eta) \frac{d s}{s} \\
& +\int_{\eta}^{\delta-\eta}(\sin n s) P\left(\frac{1}{s+\eta}\right) \phi(s+\eta) \frac{\eta d s}{s(s+\eta)} \\
& -\int_{0}^{\eta}(\sin n s) P\left(\frac{1}{s+\eta}\right) \phi(s+\eta) \frac{d s}{s+\eta} \\
& +\int_{\delta-\eta}^{\delta}(\sin n s) P\left(\frac{1}{s}\right) \phi(s) \frac{d s}{s} \\
\equiv & \sum_{k=1}^{\delta} U_{k} .
\end{aligned}
$$

The estimates of these various expressions follow standard procedure so we can restrict ourselves to a mere outline of the argument. $U_{1}$ will tend to zero after division by $P_{n}$ by virtue of (9.18). Next, for $0<\alpha<\beta$,

so that

$$
P(\beta)-P(\alpha)=\int_{\alpha}^{\beta} p(t) d t+O\{p(\alpha)\}+O\{p(\beta)\}
$$

$$
\begin{gathered}
U_{2}=O\left\{\int_{\eta}^{\delta}|\phi(s+\eta)| \frac{d s}{s} \int_{1 /(s+\eta)}^{1 / s} p(t) d t\right\}+O\left\{\int_{\eta}^{\delta}|\phi(s+\eta)| p\left(\frac{1}{s}\right) \frac{d s}{s}\right\} \\
+O\left\{\int_{\eta}^{\delta}|\phi(s+\eta)| p\left(\frac{1}{s+\eta}\right) \frac{d s}{s}\right\} .
\end{gathered}
$$


The usual method of integration by parts leads to a number of expressions each of which is almost immediately seen to be $o\left(P_{n}\right)$. The only term which causes difficulties is

$$
\int_{\eta}^{\delta} \Phi(s+\eta) \frac{d s}{s^{2}} \int_{1 /(8+\eta)}^{1 / 8} p(t) d t=o\left\{\int_{0}^{\delta} \frac{d s}{s} \int_{1 /(8+\eta)}^{1 / 8} p(t) d t\right\}=o\left(P_{n}\right)
$$

as is seen by interchanging the order of the two integrations. Further

$$
\begin{aligned}
\frac{U_{3}}{P_{n}} & =O\left\{\frac{\eta}{P_{n}} \int_{\eta}^{\delta}|\phi(s+\eta)| P\left(\frac{1}{s+\eta}\right) \frac{d s}{s(s+\eta)}\right\} \\
& =O\left\{\eta \int_{\eta}^{\delta}|\phi(s+\eta)| \frac{d s}{s^{2}}\right\}=o(1)
\end{aligned}
$$

since $x \subset E(L ; f)$. Finally $U_{4}$ and $U_{5}$ are $o(1)$, the former because $x \subset E(L ; f)$, the latter because the length of the interval of integration tends to zero. This completes the proof of Theorem $\mathrm{IV}_{3}$.

We finish the discussion of the semi-monotone case with a remark on the Lebesgue constants of the corresponding kernels $N_{n}(t)$. Lemmas 7 and 9 plus formulas (7.06), (9.07) and (9.09) prove

THEOREM V. If $p_{n}>0$ and the sequence $\left\{p_{n}\right\}$ satisfies conditions (3.01) and (3.02), then there exist positive constants $A$ and $B$ independent of $n$ such that

$$
A Q(n)<\int_{0}^{\pi}\left|N_{n}(t)\right| d t<B Q(n)
$$

where

$$
Q(n)=\frac{1}{P_{n}} \int_{1}^{n} \frac{P(u)}{u} d u
$$

We have clearly

$$
Q(n) \leqq \log n .
$$

On the other hand, if $Q_{0}(u)$ is a given logarithmico-exponential function, positive and continuous for $0<u$, which becomes infinite with $u$ and which satisfies the inequality

$$
Q_{0}^{\prime}(u)<\frac{1}{u}, u>0
$$

then we can construct an example of a definition of summability $\left(N, p_{v}\right)$ 
such that (9.19) holds with $Q(n)$ replaced by $Q_{0}(n)+\uparrow$ We have merely to take

$$
p_{0}=P_{0}(1), p_{n}=P_{0}(n+1)-P_{0}(n),
$$

where

$$
P_{0}(u)=\frac{1}{Q_{0}(u)} \exp \left\{\int_{1}^{u} \frac{d s}{s Q_{0}(s)}\right\} .
$$

The functions $P_{0}^{\prime}(u) / P_{0}(u)$ and $P_{0}^{\prime \prime}(u) / P_{0}(u)$, being logarithmico-exponential functions, are ultimately of a constant sign and monotone. The conditions on $Q_{0}(u)$ ensure that $P_{0}^{\prime}(u)$ is always positive and tends to zero as $u \rightarrow \infty$. Since $P_{0}^{\prime \prime}(u)$ ultimately keeps a constant sign, this sign must be negative. It follows that $p_{n}>0$ and ultimately monotone decreasing. Hence the sequence $\left\{p_{\nu}\right\}$ satisfies the conditions of Theorem V, and a simple calculation shows that the function $Q(n)$, computed from formula (9.20) for this choice of $\left\{p_{\nu}\right\}$, differs from the given function $Q_{0}(n)$ by a quantity which remains bounded as $n \rightarrow \infty$. Thus (9.19) holds with $Q(n)$ replaced by $Q_{0}(n)$. It is clear that the assumption that $Q_{0}(u)$ be a logarithmico-exponential function can be replaced by less stringent conditions.

10. Examples. We shall give some illustrations of the preceding results. We begin by taking

$$
p_{n}=(n+1)^{-1+\alpha}, \Re(\alpha)>0 .
$$

For real values of $\alpha$ between 0 and 1 the corresponding method $\left(N, p_{v}\right)$ is equivalent to $(C, \alpha)$. It is easily seen that the conditions of Theorem I are satisfied by this sequence, so the method is Fourier-effective.

The case $\alpha=0$ leads to a definition of summability $\left(N, p_{v}\right)$ which is not $(F)$-effective, since the sequence is monotone decreasing, but (3.03) is not satisfied. The method $\left(N,(\nu+1)^{-1}\right)$ is clearly equivalent to the method of summation defined by

$$
y_{n}=\frac{1}{\log n}\left\{\frac{x_{0}}{n+1}+\frac{x_{1}}{n}+\cdots+\frac{x_{n}}{1}\right\},
$$

called harmonic summation by M. Riesz [7]. It follows that the harmonic means do not define an $(F)$-effective method of summation. $\ddagger$ A necessary and

† Some time ago one of the authors mentioned the problem of constructing a method of summation with preassigned rate of growth of the corresponding Lebesgue constants to Dr. R. P. Agnew who then proceeded to construct methods of summation equivalent to convergence having this property.

$\ddagger$ We are indebted to Professor M. Riesz for the information that this result has already been found by one of his former pupils, Dr. N. K. A. Juringius, who, however, to the best of our knowledge, has not published any proof. 
sufficient condition that the $n$th harmonic mean of a Fourier series shall converge to $f(x)$ at an $(L)$-regular point is by Theorem III that

$$
\lim _{n \rightarrow \infty} \frac{1}{\log n} \int_{1 / n}^{\delta} \sin n t \log \frac{1}{t} \phi(t) \frac{d t}{t}=0 ;
$$

a sufficient condition is by Theorem IV

$$
\lim _{n \rightarrow \infty} \frac{1}{\log n} \int_{x / n}^{\delta}\left|\phi\left(t+\frac{\pi}{n}\right)-\phi(t)\right| \log \frac{1}{t} \frac{d t}{t}=0 .
$$

It is clear that we can also consider sequences of the form

$$
p_{n}=(n+1)^{-1+\alpha}[\log (n+e)]^{\alpha_{1}} \cdots\left[\log _{\mu}\left(n+e_{\mu}\right)\right]^{\alpha_{\mu}} .
$$

The corresponding method $\left(N, p_{v}\right)$ is found to be Fourier-effective if $\Re(\alpha)>0$, but not even $(F)$-effective if $\alpha=0$ and the other $\alpha$ 's are real.

An example of a Fourier-effective definition $\left(N, p_{v}\right)$ which satisfies (3.01) and (3.03) but not (3.02) is given by

$$
p_{\nu}=1 \text {, or 2, according as } \nu \neq m^{2} \text {, or } \nu=m^{2} \quad(m=1,2,3, \cdots) .
$$

Indeed, this definition sums any series which is summable $(C, 1)$ and whose partial sums are $o\left(n^{1 / 2}\right)$. It is well known that for the classes of trigonometric series which we are considering the partial sums are $o(\log n)$ at $\left(^{*}\right)$-regular points.

11. Applications of the relative inclusion theory. It is well known that the arithmetic means of the first order, $(C, 1)=(N, 1)=(R, 1)$, define a Fouriereffective method of summation. It follows that if a regular method of summation $A$, defined by the matrix $\mathfrak{A}=\left\|a_{m n}\right\|$, includes the method $(C, 1)$, i.e. if every series summable $(C, 1)$ is also summable $A$ to the same sum, then $A$ is also Fourier-effective.

Putting

$$
\begin{aligned}
& y_{m}=\sum_{n=0}^{m} a_{m n} x_{n}, \\
& z_{n}=\frac{1}{n+1} \sum_{k=0}^{n} x_{k},
\end{aligned}
$$

we find that the transformation from the sequence $\left\{z_{n}\right\}$ to the sequence $\left\{y_{m}\right\}$ is given by

$$
y_{m}=\sum_{n=0}^{m-1}(n+1)\left(a_{m n}-a_{m n+1}\right) z_{n}+(m+1) a_{m m} z_{m} .
$$


Applying the Silverman-Toeplitz conditions to this transformation we find that it is regular if and only if

$$
\sum_{n=0}^{m-1}(n+1)\left|a_{m n}-a_{m n+1}\right|+(m+1)\left|a_{m m}\right|<C \dagger
$$

independent of $m$, the other conditions being satisfied by virtue of the assumed regularity of $A$.

If in particular $A=\left(N, p_{v}\right)$ or $\left(R, p_{v}\right)$, condition (11.4) becomes

$$
\begin{aligned}
& n\left|p_{0}\right|+\sum_{k=1}^{n-1}(n-k)\left|p_{k}-p_{k-1}\right|<C\left|P_{n}\right|, \\
& \sum_{k=0}^{n-1}(k+1)\left|p_{k}-p_{k+1}\right|+(n+1)\left|p_{n}\right|<C\left|P_{n}\right|
\end{aligned}
$$

respectively.

Condition (11.5) is satisfied if, e.g., $\left\{p_{\nu}\right\}$ is any monotone increasing sequence subjected to the regularity condition $p_{n} / P_{n} \rightarrow 0$. Such a sequence satisfies (3.03) automatically, and (3.02) only if it also satisfies (3.01), i.e. if $n p_{n}=O\left(P_{n}\right)$. Thus we see that both (3.01) and (3.02) may be violated by a Fourier-effective method $\left(N, p_{v}\right)$.

Conditions (11.5) and (11.6) throw an interesting light on Theorem $\mathrm{II}_{1}$. We have seen that (3.03) is necessary and sufficient for Fourier-effectiveness if $p_{n}>0$ and $p_{n}$ is ultimately monotone decreasing. Such a sequence $\left\{p_{\nu}\right\}$ certainly satisfies (11.6) so that if $P_{n} \rightarrow \infty$ the method $\left(R, p_{v}\right)$ defined by the same sequence is Fourier-effective without further restrictions. In particular, $\left(N,(\nu+1)^{-1}\right)$ is not $(F)$-effective, whereas $\left(R,(\nu+1)^{-1}\right)$ is even Fourier-effective. For an application of the latter, the logarithmic means, to the summability of Fourier series, see G. H. Hardy [3], who gives reference to earlier investigations.in the field.

\section{BIBLIOGRAPHY}

1. G. C. Evans, Complements of potential theory, American Journal of Mathematics, vol. 54 (1932), pp. 213-234.

2. G. H. Hardy, Theorems relating to the summability and convergence of slowly oscillating series, Proceedings of the London Mathematical Society, (2), vol. 8 (1910), pp. 301-320.

3. G. H. Hardy, The summability of a Fourier series by logarithmic means, Quarterly Journal of Mathematics (Oxford series), vol. 2 (1931), pp. 107-112.

4. E. Hille and J. D. Tamarkin, On the summability of Fourier series, Proceedings of the National Academy of Sciences, vol. 14 (1928), pp. 915-918.

† This result, in a slightly different form, is well known; see, e.g., D.S. Morse [5], pp. 263-264, where other references are given. 
5. D. S. Morse, Relative inclusiveness of certain definitions of summability, American Journal of Mathematics, vol. 45 (1923), pp. 259-285.

6. N. E. Nörlund, Sur une application des fonctions permutables, Lunds Universitets Årsskrift, (N.F.), avd. 2, 16, No. 3 (1920); 10 pp.

7. M. Riesz, Sur l'equivalence de certaines méthodes de sommation, Proceedings of the London Mathematical Society, (2), vol. 22 (1924), pp. 412-419.

8. G. F. Woronoi, Extension of the notion of the limit of the sum of terms of an infinite series (in Russian). Proceedings of the Eleventh Congress (1901) of Russian Naturalists and Physicians, St. Petersburg, 1902, pp. 60-61. Annotated English translation by J. D. Tamarkin, Annals of Mathematics, (2), vol. 33 (1932), pp. 422-428.

\section{Princeton University,}

Princeton, N. J.

BROWN UNIVERSITY,

Providence, R. I. 